\title{
Network slicing for end-to-end latency provisioning in internet of things
}

\author{
MacHeta, Kamil; Malarski, Krzysztof Mateusz; Petersen, Martin Nordal; Ruepp, Sarah Renée
}

Published in:

Proceedings of 4th International Conference on Fog and Mobile Edge Computing

Link to article, DOI:

10.1109/fmec.2019.8795360

Publication date:

2019

Document Version

Peer reviewed version

Link back to DTU Orbit

Citation $(A P A)$ :

MacHeta, K., Malarski, K. M., Petersen, M. N., \& Ruepp, S. R. (2019). Network slicing for end-to-end latency provisioning in internet of things. In Proceedings of 4th International Conference on Fog and Mobile Edge Computing [8795360] IEEE. https://doi.org/10.1109/fmec.2019.8795360

\section{General rights}

Copyright and moral rights for the publications made accessible in the public portal are retained by the authors and/or other copyright owners and it is a condition of accessing publications that users recognise and abide by the legal requirements associated with these rights.

- Users may download and print one copy of any publication from the public portal for the purpose of private study or research.

- You may not further distribute the material or use it for any profit-making activity or commercial gain

- You may freely distribute the URL identifying the publication in the public portal 


\title{
Network Slicing for End-to-End Latency Provisioning in Internet of Things
}

\author{
Kamil Macheta, Krzysztof Mateusz Malarski, Martin Nordal Petersen, Sarah Ruepp \\ Department of Photonics Engineering \\ Technical University of Denmark, DTU \\ Kgs. Lyngby, Denmark \\ $\{$ s150763, krmal, mnpe, srru\}@fotonik.dtu.dk
}

\begin{abstract}
The Internet of Things technology is vastly growing, and more and more applications are being linked to IoT. IoT is moving from a massive deployment and gadget-like applications to also include critical applications for industry, telemedicine or utilities. In this work, we present our plans for a simulation framework that can evaluate and provide end-to-end latency bound connectivity through network slicing.
\end{abstract}

Keywords - Internet of Things, network slicing, latency, NFV

\section{INTRODUCTION}

Internet of Things is moving from gadget type and massive applications towards mission critical applications. Some of these are illustrated in figure 1, and especially the integration of IoT into applications such as telemedicine, utilities and industrial applications requires strict end-to-end latency requirements. The approach considered in this paper is to reserve some network resources through Network Slicing using Network Function Virtualisation, with the ultimate goal of providing latency guarantees to applications that require them.

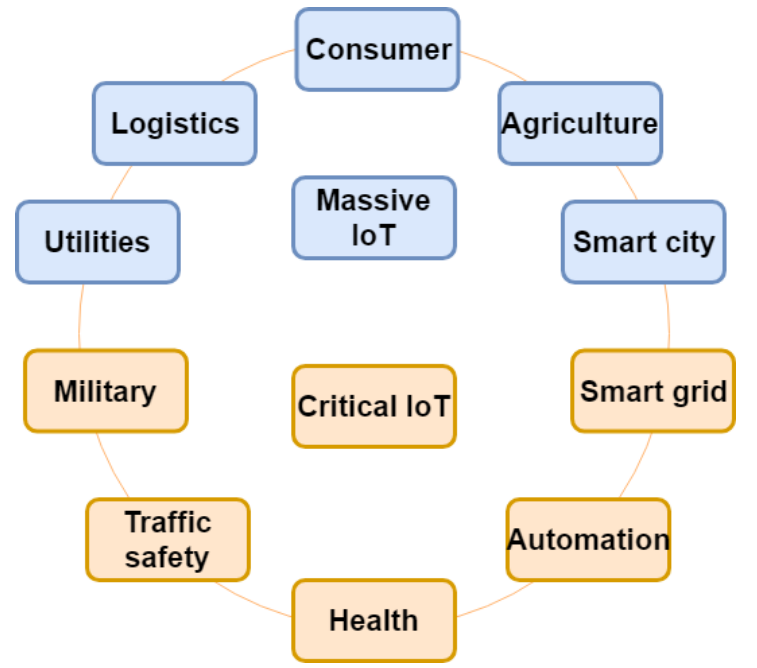

Fig. 1. Novel IoT applications

Network function virtualisation (NFV) is a relatively new approach in computer networks, which introduces virtualisation hence an abstraction into the view of the network. This leads to similar benefits as in the case of virtualisation in computer science terms. Some of the positive factors considering application of NFV can be more flexible infrastructure management, creation of networks seen as an abstract of the physical network or efficiency in resource usage.

Network Slicing is a completely new approach of creating managed vertical network slices suited for specific applications within industry branches or individuals. As a concept it is assumed to use both NFV and SDN, in order to abstract from physical network infrastructure and enable interconnection of any nodes. A network slice is a basic unit within network slicing technology. It is created by a set of functions and resources that are fulfilling purpose of the service. By definition, the network slice is independent from the physical network and any logical structures including network domains owned by separate operators. The minimal network slice consists of resources that allow providing connectivity between two entities, so a vertical protocol stack is needed. Network slicing architecture is depicted in figure 2 .

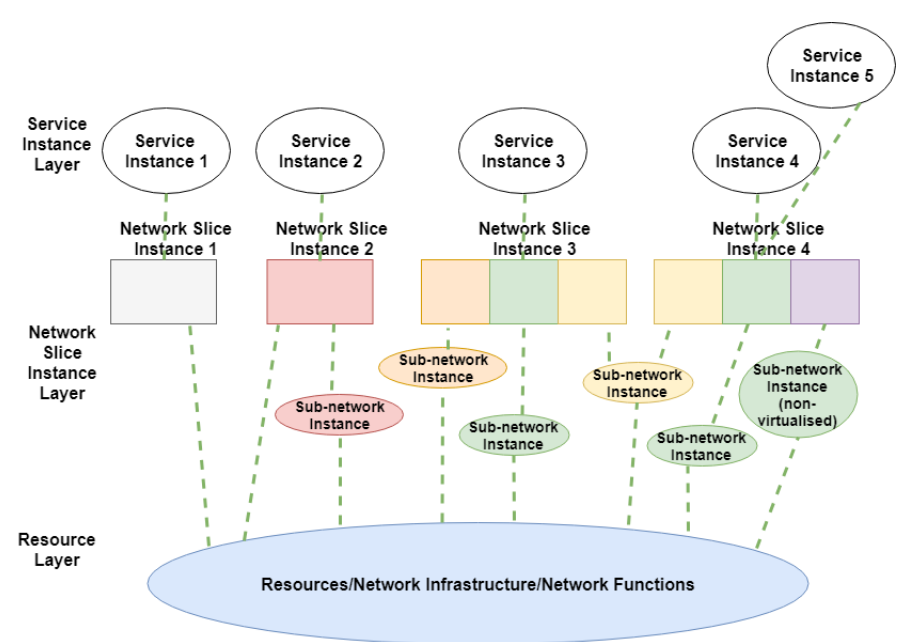

Fig. 2. Network slicing architecture

\section{SLICING FOR INTERNET OF THINGS}

It is more common to observe constantly increasing demand for services of new generation in various industry branches; the characteristics of such a service can be e.g. transmitting large amounts of data or quite the opposite, transmission of small amounts of data but with stringent latency requirements due to its real-time nature. This creates a problem of insufficient 
flexibility with assigning network resources according to the requirements.

There are several major approaches currently used or being considered as a solution for the problem. The first approach is based either on manual configuration of the devices, which requires a lot of manpower and is infeasible in the case of constantly changing requirements and rapidity of new services' deployment, especially considering constantly emerging IoT applications. The second approach is based on utilising NFV and creating verticals that are helpful in terms of resource efficiency. However, this approach is not commonly used in its pure version, since it lacks the desired level of flexibility. The major challenge in NFV is the orchestration of created network slices and automation of resource allocation process. There are also minor implementation specific problems, such as the way the resources are virtualised or minimal slice resources.

The network slicing differs from classical computer networks in terms of flexibility of resource configuration as well as the network management. Network organised as slices are separate verticals that might consist of nodes administered by different operators and being assigned to different network domains. That leads to the issue of resource orchestration. There has been a couple of architecture proposals that might be a solution for the problem. 5G!Pagoda [1] proposes Multi-domain Orchestration Architecture which consists of two major entities: 1) Domain specific slice orchestrator which is responsible for management of nodes within one network domain and 2) multidomain slice orchestrator that coordinates inter-domain transactions. The document also defines a complete infrastructure of network slicing for $5 \mathrm{G}$ including approaches to minimize resources over-provisioning in the case of single slices. The idea is to divide and virtualise current EPC into the set of micro functions. Then, the minimal setup that allows connectivity might be defined as Lightweight Core Network and additional features might be added depending on requirements of the application served.

Another architecture example is DASMO [2], which extends current ETSI MANO [3] standard. DASMO, similarly as in 5G!Pagoda approach, provides slide management but divided into three separate planes: Application, Control and Management, which are managed by an interface familiar to the end user through Slice Manager. The inter-domain part of the management is handled by OSS/BSS. Management (granting) physical network resources is handled by NFVO. Apart from the architectural approaches to the problem there is a set of different requirements that $5 \mathrm{G}$ network have to fulfil. Most of them were accurately described by NGMN in $5 \mathrm{G}$ Whitepaper [4].

The planned method of achieving low end-to-end latency slices is creation of a network model that will portray network slicing for specified application to the highest possible extent.

There will be possibility of a simulation scenario for latency requirements of one IoT application, e.g. telemedical or industrial use cases. The model should be able to provide creation of NFV verticals, network slices and methods for their centralized orchestration. In terms of communication frameworks, LP-WAN technologies presented in figure 3 will be implemented to cover long-range applications, and to evaluate their end-to-end latency performance. Using NS3 modelling software, the model will be extended upon existing libraries, such as LoRaWAN.[5]

The limitation of our simulation framework comes from the fact that at a time only one IoT application can be handled. Moreover, the scope of network slicing is narrowed down to latency provisioning. Nevertheless, we consider broadening the functionality of the simulator once the proof-of-concept implementation of the primary idea is developed.

\section{LONG DISTANCE (km)}

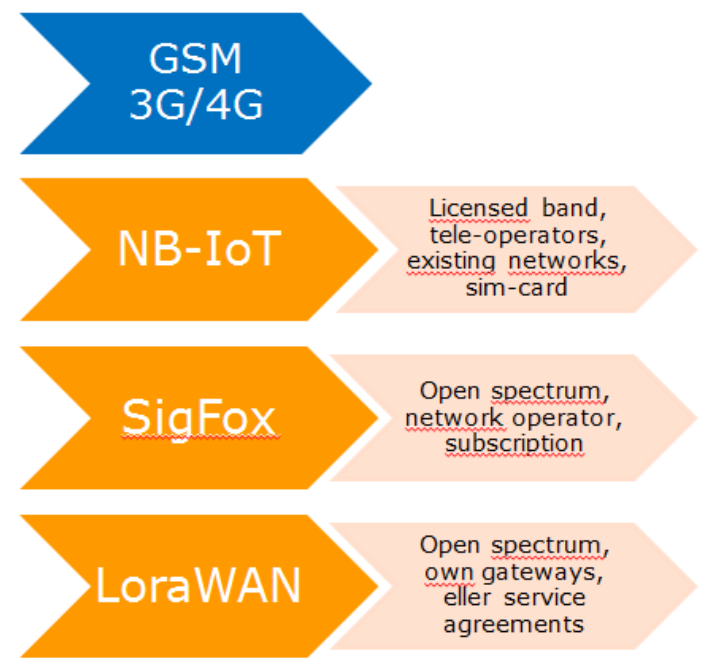

Fig. 3. Long-range IoT communication technologies

\section{CONCLUSION}

In this paper, we present our plans for a simulation framework, which can evaluate and provide end-to-end latency bound connectivity through network slicing. The simulation framework can be used to evaluate different LP-WAN technologies' suitability for various latency dependent use cases.

\section{ACKNOWLEDGMENT}

This work has been partially supported by the Danish Innovation Foundation and Eureka Turbo through the Watch4Life project.

\section{REFERENCES}

[1] FOKUS, AALTO, Ericsson, Orange, Waseda U, EURECOM, MI, NESIC 5G! Pagoda. August 2017.

[2] Sławomir Kukliński, Lechosław Tomaszewski DASMO: A Scalable Approach to Network Slices Management and Orchestration.

[3] ETSI Network Functions Virtualisation (NFV); Management and Orchestration. ETSI GS NFV-MAN 001 V1.1.1 2014-12.

[4] NGMN Alliance. NGMN 5G White Paper. 2015-01.

[5] LoRaWAN model. https://github.com/imec-idlab/ns-3-devgit/tree/lorawan 